\title{
Efficacy of Allogenic Bone Graft Per Se and when Augmented with Various Adjuvants in Fracture Healing
}

\author{
Lakhwani OP ${ }^{1}$, Jindal $\mathbf{M}^{2 *}$, Agarwal ${ }^{1}$ and Garg Keerty ${ }^{3}$ \\ ${ }^{1}$ Department of Orthopaedics, ESI - PGIMSR, India \\ ${ }^{2}$ Department of Orthopaedics, Kalpana Chawla Govt Medical College, India \\ ${ }^{3}$ Department of Anaesthesia, Guru Nanak Medical College, India
}

Submission: March 28, 2018; Published: May 04, 2018

*Corresponding author: Jindal M, Department of Orthopaedics, Assistant Professor, Kalpana Chawla Govt Medical College, Karnal, Haryana, India, Tel: +91-9891904545; Email: mohitjindal2006@gmail.com

\begin{abstract}
Introduction: Bone grafts are often required in current orthopedic practice. Limited availability and donor site morbidity associated with the use of autograft has lead to the use of allogenic bone graft. However use of allografts may carry a potential risk of infection and disease transmission and has less osteogenic potential compared to autografts. Hence use of adjuvants such as autologous bone marrow and bone graft along with allogenic bone is required and formed the basis of this study.
\end{abstract}

Materials and Methods: Study involved case series of eighteen patients with difficult fracture and established delayed \& non union treated with allogenic bone graft alone or with adjuvants (bone marrow and or autograft). Outcome was assessed in terms of fracture healing, infection and osteointegration.

Results: over all 15 fractures (83\%) united. None of the patient developed infection. Three cases, of which two where allogenic bone graft was used alone and one in which allograft augmented with autologous bone marrow was used, failed to unite and bone graft was absorbed gradually.

Conclusion: Allogenic bone can serve as excellent material for prophylactic or primary bone grafting in difficult to heal fractures as it potentiates bone healing and bridges bone gaps. However in established cases of delayed and non unions which require grafts with good osteogenic potential, use of allogenic grafts along with adjuvants like bone marrow and autologous bone graft is more beneficial.

Level of Evidence: level IV.

Keywords: Allogenic bone graft; Fracture healing; Non union; Difficult fracture

Introduction

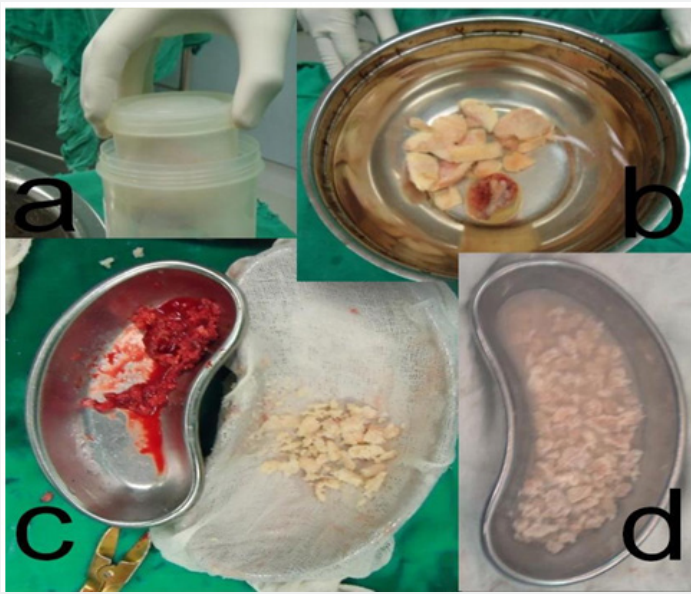

Figure 1: showing intra operative processing of Allograft obtained from institutionalized bone bank

a. Graft taken from the double containers, as stored in Bone bank.

b. Graft morcelized into fragments of $0.5-0.6 \mathrm{~cm}$ in size.

c. Washed morcelized graft in a kidney tray.

d. Graft mixed with vancomycin and sterile water so as to make a concentration of $50 \mathrm{mg} / \mathrm{ml}$. 
Bone grafts1 are often necessary to potentiate the bone healing in cases of fractures having communition, bone loss, delayed union, non unions and other difficult to heal fractures $[1,2]$. Autologous bone grafts are the gold standard $[3,4]$ due to their higher osteoinductive potential as compared to allografts and no risk of immunogenic reaction and disease transmission [3-5]. But autologous bone grafts have restrictions of limited availability and donor site morbidity [5,6] Allogenic bone grafts, on the other hand, are available in sufficient quantities and without any risk of donor site morbidity [5,6]. but their effectiveness in fracture healing is still debatable [6-8]. Present case series describe the use of allogenic bone graft alone and combined with adjuvants like autologous bone graft and/or autologous bone marrow (Figure 1). Improved technique of Gamma sterilization and prophylactic vancomycin impregnation were also used to take care of allograft associated infection $[9,10]$. Results were assessed in terms of fracture healing, osteointegartion and infection.

\section{Material and Methods}

The study was approved by institutional ethical committee of ESI-PGIMSR, Basaidarapur, New Delhi and all patients were fully informed and agreed to participate in the study. The inclusion criteria were patients with difficult to heal fracture such as comminuted fractures, fractures with bone defects/ bone loss, delayed union and non unions. Exclusion criteria were active osteoarticular infection at the concerned site. From 2011 to 2013 eighteen consecutive cases of selected fractures were treated with allogenic bone grafting with appropriate surgical procedure for fixation wherever required. Out of eighteen cases, nine cases were of established non-unions, four cases were of delayed union and rest five cases belonged to difficult fracture category, that is, relatively fresh fractures with severe communition and bone loss. In study among 18 patients, allogenic bone graft augmented with autologous bone graft was used in nine patients ( $\mathrm{n} 1=9$ ), autologous bone marrow in five $(n 2=5)$ and allograft alone in four $(n 3=4)$. The case series consisted of fifteen male patients and three female patients with a mean age of 35 years (16-68 years) and a mean follow-up of 12 months (8 months- 17 months).

\section{Graft Preparation}

Allogenic bone grafts procured from consenting patients having undergone hip and knee arthroplasty at institutional bone bank. Allografts were stored at $-80^{\circ} \mathrm{C}$ for minimum of three months before use. The terminal Sterilization was achieved by giving a dose of $25 \mathrm{~K}$ grays of radiation at an accredited centre. The graft was manually morcelized to fragment size of 0.4 $0.6 \mathrm{cms}$ and washed with plenty of sterile saline. The bacterial cultures of the allograft were taken before implantation to ascertain sterility and were all negative. Morcellized bone grafts were impregnated with solution of vancomycin $50 \mathrm{mg} / \mathrm{ml}$ as described by Witso et al. [10] for a period of one hour.

Patients were seen at $3^{\text {rd }}$ wound check, then 2 weeks postoperatively for suture removal and again at 6 and 12 weeks. Particular attention was given to any sign of infection/ inflammation. Radiographs were done every four weeks for initial four months and then at every two months till completion of follow up. Radiographs were used to qualitatively determine the status of union which was defined as presence of at least three of four healed cortices and crossing trabeculae on anteroposterior and lateral radiographs while clinical healing was defined as the absence of functional pain and local tenderness at fracture site. Rate of osteointegration of allograft was assessed using criteria given by Sloof et al. [11] (Table 1) and infection as per clinical, haematological and biochemistry test according to the Surgical Site Infection (SSI) guidelines.

Table 1: Observation Table.

\begin{tabular}{|c|c|c|c|c|c|c|c|c|c|}
\hline $\begin{array}{c}\text { Patient } \\
\text { serial no. }\end{array}$ & $\begin{array}{l}\text { Age/ } \\
\text { Gender }\end{array}$ & $\begin{array}{c}\text { Insurance } \\
\text { no. }\end{array}$ & Diagnosis & $\begin{array}{c}\text { Surgical } \\
\text { procedure }\end{array}$ & $\begin{array}{c}\text { Vanco } \\
\text { mycin } \\
\text { impreg } \\
\text { nation }\end{array}$ & $\begin{array}{c}\text { Follow up } \\
\text { period } \\
\text { (month) }\end{array}$ & $\begin{array}{l}\text { Osteointeg } \\
\text { ration (Sloof's } \\
\text { criteria) }\end{array}$ & $\begin{array}{l}\text { Healing } \\
\text { status of } \\
\text { bone(Neer,s } \\
\text { criteria for } \\
\text { lytic lesions } \\
\text { and union } \\
\text { status for } \\
\text { fractures) }\end{array}$ & Infection \\
\hline 1 & $39 / F$ & 1112700550 & $\begin{array}{l}\text { Spondylo } \\
\text { listhesis } \\
\text { L4 over L5 } \\
\text { grade } 2 \text { with } \\
\text { paraparesis } \\
\text { with bowel } \\
\text { bladder } \\
\text { involvement }\end{array}$ & $\begin{array}{l}\text { Pedicle screw } \\
\text { fixation in } \\
\text { L4,L5, S1 with } \\
\text { posterior } \\
\text { fusion } \\
\text { augmented } \\
\text { with allogenic } \\
\text { bone graft }\end{array}$ & + & 18 & $\begin{array}{c}\text { Graft resorption } \\
\text { seen at } 3 \\
\text { months }\end{array}$ & $\begin{array}{l}\text { Adequate } \\
\text { fusion } \\
\text { achieved at } \\
5 \text { months }\end{array}$ & $\begin{array}{c}\text { Sterile } \\
\text { discharge } \\
\text { seen at } \\
5 \text { days } \\
\text { postop } \\
\text { erative }\end{array}$ \\
\hline 2 & $31 / \mathrm{M}$ & 2416166476 & $\begin{array}{l}\text { Delayed } \\
\text { union rt } \\
\text { radius/ulna }\end{array}$ & $\begin{array}{l}\text { ORIF plate/ } \\
\text { square nail } \\
\text { with allogenic } \\
\text { bone graft }\end{array}$ & + & 17 & $\begin{array}{c}\text { Adequate } \\
\text { osteointe } \\
\text { gration in } \\
\text { ulna seen at } 5 \\
\text { months. Partial } \\
\text { osteointe } \\
\text { gration seen in } \\
\text { radius }\end{array}$ & $\begin{array}{l}\text { Ulnar } \\
\text { fracture } \\
\text { union at } \\
5 \text { months. } \\
\text { Radius } \\
\text { nonunion }\end{array}$ & Negative \\
\hline
\end{tabular}


Orthopedics and Rheumatology Open Access Journal (OROAJ)

\begin{tabular}{|c|c|c|c|c|c|c|c|c|c|}
\hline 3 & $40 / \mathrm{F}$ & 1504678179 & $\begin{array}{l}\text { Anterolis } \\
\text { thesis L4 } \\
\text { over L5 }\end{array}$ & $\begin{array}{l}\text { Pedicle screw } \\
\text { fixation } \\
\text { L4,L5,SI } \\
\text { posteror } \\
\text { fusion with } \\
\text { allogenic bone } \\
\text { graft }\end{array}$ & - & 17 & $\begin{array}{c}\text { Graft resorption } \\
\text { seen at } 3 \\
\text { months }\end{array}$ & $\begin{array}{l}\text { Adequate } \\
\text { fusion } \\
\text { achieved at } \\
5 \text { months }\end{array}$ & Negative \\
\hline 4 & $52 / \mathrm{M}$ & 6709471741 & $\begin{array}{l}\text { Delayed } \\
\text { intertro } \\
\text { chanteric } \\
\text { fracture left } \\
\text { side with } \\
\text { PFN in situ }\end{array}$ & $\begin{array}{l}\text { Allograft } \\
\text { placement in } \\
\text { region of bone } \\
\text { loss. }\end{array}$ & - & 16 & $\begin{array}{c}\text { Adequate } \\
\text { osteointe } \\
\text { gration seen at } 5 \\
\text { months. }\end{array}$ & $\begin{array}{c}\text { Fracture } \\
\text { union seen } \\
\text { at around } 8 \\
\text { months }\end{array}$ & Negative \\
\hline 5 & $25 / \mathrm{M}$ & 1112582312 & $\begin{array}{l}\text { Aneurysmal } \\
\text { bone cyst } \\
\text { right talus }\end{array}$ & $\begin{array}{c}\text { Curettage } \\
\text { with allogenic } \\
\text { bone graft }\end{array}$ & - & 16 & $\begin{array}{l}\text { Adequate } \\
\text { osteoin } \\
\text { tegration seen at } \\
6 \text { months. }\end{array}$ & $\begin{array}{l}\text { Partial } \\
\text { healing } \\
\text { stage ii of } \\
\text { Neer's }\end{array}$ & $\begin{array}{c}\text { Pseudo } \\
\text { monas } \\
\text { aerugi } \\
\text { nosa } \\
\text { positive } \\
\text { serous } \\
\text { discharge } \\
\text { on 5th } \\
\text { day. } \\
\text { Resolved } \\
\text { with } \\
\text { medic } \\
\text { ation. }\end{array}$ \\
\hline 6 & $36 / \mathrm{M}$ & 1113520202 & $\begin{array}{l}\text { \# Nonunion } \\
\text { shaft femur } \\
\text { rt side with } \\
\text { ILN in situ }\end{array}$ & $\begin{array}{l}\text { Allograft } \\
\text { placement in } \\
\text { region of bone } \\
\text { loss }\end{array}$ & - & 16 & $\begin{array}{c}\text { Adequate } \\
\text { osteointeg- } \\
\text { ration seen at } 6 \\
\text { months. }\end{array}$ & $\begin{array}{c}\text { Fracture } \\
\text { union seen } \\
\text { at around } 6 \\
\text { months }\end{array}$ & Negative \\
\hline 7 & $65 / M$ & 6913319130 & $\begin{array}{c}\text { \# Suprac } \\
\text { ondylar } \\
\text { femur } \\
\text { fracture rt } \\
\text { side (Difficult } \\
\text { \#) }\end{array}$ & $\begin{array}{l}\text { ORIF with LCP } \\
\text { with allogenic } \\
\text { and autogenic } \\
\text { bone grafting }\end{array}$ & + & 15 & $\begin{array}{c}\text { Adequate } \\
\text { osteointe } \\
\text { gration seen at } 8 \\
\text { months }\end{array}$ & $\begin{array}{c}\text { Fracture } \\
\text { union seen } \\
\text { at around } 8 \\
\text { months }\end{array}$ & Negative \\
\hline 8 & $66 / F$ & 1307555416 & $\begin{array}{c}\text { \# Shaft } \\
\text { femur rt side } \\
\text { with broken } \\
\text { interlocking } \\
\text { nail in } \\
\text { situ(Difficult } \\
\text { \#) }\end{array}$ & $\begin{array}{c}\text { Exchange } \\
\text { nailing with } \\
\text { allogenic bone } \\
\text { graft }\end{array}$ & - & 15 & $\begin{array}{c}\text { Adequate } \\
\text { osteointe } \\
\text { gration seen at } 8 \\
\text { months }\end{array}$ & $\begin{array}{c}\text { Fracture } \\
\text { union seen } \\
\text { at around } 8 \\
\text { months }\end{array}$ & Negative \\
\hline 9 & $47 / \mathrm{M}$ & 2203427230 & $\begin{array}{l}\text { Delayed } \\
\text { union \# shaft } \\
\text { femur rt side }\end{array}$ & $\begin{array}{l}\text { Allogenic } \\
\text { bone graft } \\
\text { placement at } \\
\text { site of bone } \\
\text { loss }\end{array}$ & + & 14 & $\begin{array}{c}\text { Adequate } \\
\text { osteointe } \\
\text { gration seen at } 8 \\
\text { months }\end{array}$ & $\begin{array}{c}\text { Fracture } \\
\text { union seen } \\
\text { at around } 8 \\
\text { months }\end{array}$ & Negative \\
\hline 10 & $36 / \mathrm{M}$ & 1113072964 & $\begin{array}{l}\text { Giant Cell } \\
\text { Tumour } \\
\text { right } \\
\text { proximal } \\
\text { tibia }\end{array}$ & $\begin{array}{l}\text { Curettage with } \\
\text { allogenic bone } \\
\text { graft }\end{array}$ & - & 14 & $\begin{array}{c}\text { Adequate } \\
\text { osteointe } \\
\text { gration seen at } 7 \\
\text { months }\end{array}$ & $\begin{array}{l}\text { Complete } \\
\text { healing } \\
\text { neer's } \\
\text { stage1 seen } \\
\text { at around } 7 \\
\text { months }\end{array}$ & Negative \\
\hline 11 & $14 / \mathrm{M}$ & 2213527252 & $\begin{array}{l}\text { Simple Bone } \\
\text { Cyst right } \\
\text { proximal } \\
\text { humerus }\end{array}$ & $\begin{array}{l}\text { curettage } \\
\text { with allogenic } \\
\text { bone graft } \\
\text { with internal } \\
\text { fixation with } \\
\text { TENS nailing }\end{array}$ & - & 14 & $\begin{array}{c}\text { Adequate } \\
\text { osteointe } \\
\text { gration seen at } 3 \\
\text { months }\end{array}$ & $\begin{array}{l}\text { Complete } \\
\text { healing } \\
\text { neer's stage } \\
1 \text { seen at } \\
\text { around } 3 \\
\text { months }\end{array}$ & Negative \\
\hline
\end{tabular}


Orthopedics and Rheumatology Open Access Journal (OROAJ)

\begin{tabular}{|c|c|c|c|c|c|c|c|c|c|}
\hline 12 & $25 / \mathrm{F}$ & 2014268233 & $\begin{array}{l}\text { Aneurysmal } \\
\text { Bone Cyst } \\
\text { Rt proximal } \\
\text { femur }\end{array}$ & $\begin{array}{c}\text { Curettage } \\
\text { with allogenic } \\
\text { and autogenic } \\
\text { bone graft } \\
\text { with fixation } \\
\text { using } \\
\text { proximal } \\
\text { femoral nail. }\end{array}$ & + & 14 & $\begin{array}{c}\text { Adequate } \\
\text { osteointe } \\
\text { gration seen at } 8 \\
\text { months }\end{array}$ & $\begin{array}{l}\text { Complete } \\
\text { healing } \\
\text { neer's stage } \\
1 \text { seen at } \\
\text { around } 8 \\
\text { months }\end{array}$ & Negative \\
\hline 13 & $26 / \mathrm{F}$ & 1113663596 & $\begin{array}{l}\text { Simple Bone } \\
\text { Cyst distal } \\
\text { end of radius }\end{array}$ & $\begin{array}{l}\text { Curettage } \\
\text { with allogenic } \\
\text { bone graft } \\
\text { with internal } \\
\text { fixation with } \\
\text { distal end } \\
\text { radius locking } \\
\text { compression } \\
\text { plate }\end{array}$ & + & 13 & $\begin{array}{c}\text { Adequate } \\
\text { osteointe } \\
\text { gration seen at } 5 \\
\text { months }\end{array}$ & $\begin{array}{l}\text { Complete } \\
\text { healing } \\
\text { neer's stage } \\
1 \text { seen at } \\
\text { around } 5 \\
\text { months }\end{array}$ & Negative \\
\hline 14 & $43 / \mathrm{M}$ & 6709774599 & $\begin{array}{l}\text { Giant Cell } \\
\text { Tumour left } \\
\text { proximal } \\
\text { tibia }\end{array}$ & $\begin{array}{l}\text { Curettage with } \\
\text { burring with } \\
\text { allogenic bone } \\
\text { graft }\end{array}$ & + & 13 & $\begin{array}{c}\text { Adequate } \\
\text { osteointe } \\
\text { gration seen at } 8 \\
\text { months }\end{array}$ & $\begin{array}{l}\text { Complete } \\
\text { healing } \\
\text { neer's stage } \\
1 \text { seen at } \\
\text { around } 8 \\
\text { months }\end{array}$ & Negative \\
\hline 15 & $64 / F$ & 1504660752 & $\begin{array}{l}\text { Non union \# } \\
\text { intertro } \\
\text { chanteric (1 } \\
\text { year old) }\end{array}$ & $\begin{array}{c}\text { Allogenic } \\
\text { bone graft } \\
\text { with internal } \\
\text { fixation with } \\
\text { PFN and TBW }\end{array}$ & - & 12 & $\begin{array}{c}\text { Adequate } \\
\text { osteointe } \\
\text { gration seen at } 7 \\
\text { months }\end{array}$ & $\begin{array}{c}\text { Fracture } \\
\text { union seen } \\
\text { at around } 7 \\
\text { months }\end{array}$ & Negative \\
\hline 16 & $60 / \mathrm{M}$ & 1013626926 & $\begin{array}{l}\text { Non union } \\
\text { Subtroc } \\
\text { hanteric \# } 5 \\
\text { months old }\end{array}$ & $\begin{array}{l}\text { Allogenic and } \\
\text { autogenic } \\
\text { bone graft } \\
\text { with internal } \\
\text { fixation with } 8 \\
\text { hole DCS }\end{array}$ & + & 11 & $\begin{array}{c}\text { Adequate } \\
\text { osteointe } \\
\text { gration seen at } 8 \\
\text { months }\end{array}$ & $\begin{array}{c}\text { Fracture } \\
\text { union seen } \\
\text { at around } 8 \\
\text { months }\end{array}$ & Negative \\
\hline 17 & $41 / \mathrm{M}$ & 2205803905 & $\begin{array}{l}\text { Non union \# } \\
\text { subtroc } \\
\text { hanteric } \\
\text { femur right } \\
\text { side with } \\
\text { DHS implant } \\
\text { in situ. }\end{array}$ & $\begin{array}{l}\text { Allogenic and } \\
\text { autogenic } \\
\text { bone grafting } \\
\text { at site of bone } \\
\text { loss. }\end{array}$ & - & 11 & $\begin{array}{c}\text { Adequate } \\
\text { osteointe } \\
\text { gration seen at } 8 \\
\text { months }\end{array}$ & $\begin{array}{c}\text { Fracture } \\
\text { union seen } \\
\text { at around } 8 \\
\text { months }\end{array}$ & Negative \\
\hline 18 & $24 / F$ & 1111901549 & $\begin{array}{l}\text { Giant cell } \\
\text { tumour right } \\
\text { proximal } \\
\text { tibia }\end{array}$ & $\begin{array}{l}\text { Curettage with } \\
\text { burring with } \\
\text { allogenic bone } \\
\text { grafting }\end{array}$ & + & 11 & $\begin{array}{c}\text { Adequate } \\
\text { osteointe } \\
\text { gration seen at } 9 \\
\text { months }\end{array}$ & $\begin{array}{c}\text { Fracture } \\
\text { union seen } \\
\text { at around } 9 \\
\text { months }\end{array}$ & Negative \\
\hline 19 & $30 / \mathrm{M}$ & 1112485971 & $\begin{array}{c}\text { Open } \\
\text { segmental } \\
\text { fracture tibia } \\
\text { midshaft left } \\
\text { side }\end{array}$ & $\begin{array}{c}\text { Allogenic } \\
\text { bone graft } \\
\text { with fixation } \\
\text { with ILN with } \\
\text { rotation flap. }\end{array}$ & + & 10 & $\begin{array}{c}\text { Partial osteointe } \\
\text { gration seen at } 8 \\
\text { months }\end{array}$ & $\begin{array}{l}\text { Incomplete } \\
\text { union seen } \\
\text { at around } 8 \\
\text { months }\end{array}$ & Negative \\
\hline 20 & $47 / F$ & 1314438539 & $\begin{array}{c}\text { Gap } \\
\text { nonunion } \\
\text { \# shaft } \\
\text { humerus } \\
\text { midshaft } \\
\text { with LCP in } \\
\text { situ. }\end{array}$ & $\begin{array}{l}\text { Implant } \\
\text { removal with } \\
\text { allogenic bone } \\
\text { graft with } \\
\text { fixation with } 9 \\
\text { hole LCP with } \\
\text { fibular strut } \\
\text { graft }\end{array}$ & + & 9 & $\begin{array}{c}\text { Adequate } \\
\text { osteointegration } \\
\text { seen at } 8 \\
\text { months }\end{array}$ & $\begin{array}{c}\text { Fracture } \\
\text { union seen } \\
\text { at around } 8 \\
\text { months }\end{array}$ & Negative \\
\hline 21 & $28 / \mathrm{M}$ & 2213275293 & $\begin{array}{l}\text { Delayed } \\
\text { union \# } \\
\text { femur } \\
\text { midshaft lt } \\
\text { side }\end{array}$ & $\begin{array}{l}\text { Allogenic and } \\
\text { autogenic } \\
\text { bone grafting } \\
\text { with fixation } \\
\text { with K nail }\end{array}$ & + & 9 & $\begin{array}{c}\text { Adequate } \\
\text { osteointe } \\
\text { gration seen at } 8 \\
\text { months }\end{array}$ & $\begin{array}{c}\text { Fracture } \\
\text { union seen } \\
\text { at around } 8 \\
\text { months }\end{array}$ & Negative \\
\hline
\end{tabular}


Orthopedics and Rheumatology Open Access Journal (OROAJ)

\begin{tabular}{|c|c|c|c|c|c|c|c|c|c|}
\hline 22 & $25 / \mathrm{M}$ & 1312523535 & $\begin{array}{l}\text { \# Non union } \\
\text { rt femur } \\
\text { midshaft } \\
\text { with ILN in } \\
\text { situ }\end{array}$ & $\begin{array}{c}\text { Allogenic } \\
\text { bone grafting } \\
\text { with exchange } \\
\text { nailing }\end{array}$ & + & 9 & $\begin{array}{c}\text { Adequate } \\
\text { osteointegration } \\
\text { seen at } 8 \\
\text { months }\end{array}$ & $\begin{array}{l}\text { Fracture } \\
\text { union seen } \\
\text { at around } 8 \\
\text { months }\end{array}$ & Negative \\
\hline 23 & $43 / \mathrm{M}$ & 1504204920 & $\begin{array}{l}\text { \# Nonunion } \\
\text { shaft femur } \\
\text { left side with } \\
\text { failed LCP }\end{array}$ & $\begin{array}{c}\text { Allogenic and } \\
\text { autogenic } \\
\text { bone graft } \\
\text { with internal } \\
\text { fixation with } \\
\text { LCP }\end{array}$ & - & 8 & $\begin{array}{c}\text { Adequate } \\
\text { osteointe } \\
\text { gration seen at } 9 \\
\text { months }\end{array}$ & $\begin{array}{l}\text { Fracture } \\
\text { union seen } \\
\text { at around } 9 \\
\text { months }\end{array}$ & Negative \\
\hline 24 & $50 / \mathrm{M}$ & 1114022762 & $\begin{array}{c}\text { Rt. Side } \\
\text { femur \# } \\
\text { suprac } \\
\text { ondylar with } \\
\text { interco } \\
\text { ndylar } \\
\text { extension } \\
\text { right side } \\
\text { (Difficult } \\
\text { fracture) }\end{array}$ & $\begin{array}{l}\text { Allogenic and } \\
\text { autogenic } \\
\text { bone graft } \\
\text { with fixation } \\
\text { with ilizarov }\end{array}$ & - & 8 & $\begin{array}{c}\text { Adequate } \\
\text { osteointe } \\
\text { gration seen at } 7 \\
\text { months }\end{array}$ & $\begin{array}{l}\text { Fracture } \\
\text { union seen } \\
\text { at around } 7 \\
\text { months }\end{array}$ & Negative \\
\hline 25 & $31 / \mathrm{M}$ & 1113279793 & $\begin{array}{c}\text { Atrophic } \\
\text { nonunion } \\
\text { midshaft } \\
\text { tibia \# rt side } \\
\text { with ilizarov } \\
\text { fixator in situ }\end{array}$ & $\begin{array}{l}\text { Corticotomy } \\
\text { distal third } \\
\text { tibia with } \\
\text { ilizarov fixator } \\
\text { with allogenic } \\
\text { bone graft }\end{array}$ & + & 8 & $\begin{array}{c}\text { Graft resorption } \\
\text { seen at around } 5 \\
\text { months }\end{array}$ & $\begin{array}{l}\text { Fracture } \\
\text { tibia non- } \\
\text { union }\end{array}$ & Negative \\
\hline 26 & $52 / \mathrm{F}$ & 2001056939 & $\begin{array}{l}\text { Spondylo } \\
\text { listhesis L5 } \\
\text { over S1 }\end{array}$ & $\begin{array}{l}\text { Pedicle screw } \\
\text { fixation in } \\
\text { L4,L5, S1 with } \\
\text { posterior } \\
\text { fusion }\end{array}$ & - & 8 & $\begin{array}{l}\text { Graft resorption } \\
\text { seen at } 3 \\
\text { months }\end{array}$ & $\begin{array}{l}\text { Adequate } \\
\text { fusion } \\
\text { achieved at } \\
5 \text { months }\end{array}$ & Negative \\
\hline 27 & $34 / \mathrm{M}$ & 6907908300 & $\begin{array}{c}\text { Spacer } \\
\text { placed in left } \\
\text { hip following } \\
\text { removal } \\
\text { of THR } \\
\text { prosthesis }\end{array}$ & $\begin{array}{c}\text { Revision } \\
\text { THR with } \\
\text { acetabular } \\
\text { reconst } \\
\text { ruction with } \\
\text { allogenic bone } \\
\text { graft }\end{array}$ & - & 8 & $\begin{array}{l}\text { Adequate } \\
\text { osteointegration } \\
\text { of graft at } \\
\text { around } 6 \\
\text { months }\end{array}$ & $\begin{array}{l}\text { Adequate } \\
\text { fusion at } 6 \\
\text { months }\end{array}$ & Negative \\
\hline 28 & $20 / \mathrm{M}$ & 131359305 & $\begin{array}{l}\text { Aneurysmal } \\
\text { Bone Cyst } \\
\text { right radius }\end{array}$ & $\begin{array}{l}\text { Curettage with } \\
\text { allogenic bone } \\
\text { graft }\end{array}$ & + & 6 & $\begin{array}{c}\text { Adequate } \\
\text { osteointe } \\
\text { gration seen at } 6 \\
\text { months }\end{array}$ & $\begin{array}{l}\text { Complete } \\
\text { healing } \\
\text { neer's stage } \\
1 \text { seen at } \\
\text { around } 6 \\
\text { months }\end{array}$ & Negative \\
\hline 29 & $30 / \mathrm{M}$ & 221169762 & $\begin{array}{c}\text { Open } \\
\text { segmental } \\
\text { \# midshaft } \\
\text { tibia Lt. } \\
\text { side(Difficult } \\
\text { fracture) }\end{array}$ & $\begin{array}{l}\text { ILN tibia with } \\
\text { rotation flap } \\
\text { with allogenic } \\
\text { and autogenic } \\
\text { bone graft }\end{array}$ & - & 6 & $\begin{array}{c}\text { Adequate } \\
\text { osteointe } \\
\text { gration seen at } 8 \\
\text { months }\end{array}$ & $\begin{array}{l}\text { Fracture } \\
\text { union seen } \\
\text { at around } 8 \\
\text { months }\end{array}$ & Negative \\
\hline 30 & $80 / \mathrm{M}$ & 6712737859 & $\begin{array}{c}2 \text { months old } \\
\text { Neglected \# } \\
\text { intertro } \\
\text { chanter left } \\
\text { side (Difficult } \\
\text { fracture) }\end{array}$ & $\begin{array}{l}\text { PFN with } \\
\text { allogenic bone } \\
\text { graft }\end{array}$ & - & 6 & $\begin{array}{c}\text { Adequate } \\
\text { osteointe } \\
\text { gration seen at } 7 \\
\text { months }\end{array}$ & $\begin{array}{l}\text { Fracture } \\
\text { union seen } \\
\text { at around } 7 \\
\text { months }\end{array}$ & Negative \\
\hline
\end{tabular}

\section{Results}

Radiographic evaluation demonstrated osseous union following the index surgery in 15 out of the 18 cases (83\%). Time duration to achieve adequate union in nine cases where auto graft was used as adjuvant was $8 \pm 0.76$ months compared to bone marrow aspirate $9 \pm 0.49$ months and in one allograft alone case was 8.5 months. Three failure cases comprised of two cases where allograft alone were used and one case allograft with bone marrow. In all case of fracture union gradual and partial osteointegration was achieved comparing quantity of graft used in serial skiagram. In three cases of failure allogenic bone graft was gradually and completely reabsorbed by 4-5 months. None 


\section{Orthopedics and Rheumatology Open Access Journal (OROAJ)}

of the patients developed infection at the surgical site following the operative procedure during follow up period.

\section{Discussion}

The efficacy of autologous bone grafting in augmentation of fracture healing is well proven and also no risk of transmission of occult disease or infection. Various studies have reported varied success with allogenic bone graft. Hierholzer et al. [12] reported an overall union rate of $100 \%$ with use of allogenic bone graft for fracture non unions. Michael A Flierl et al. [13] compared the use of autogenic and allogenic bone graft showed that the cases with autograft took less time to union (198 $\pm 172-$ 225 days) as compared to allograft (416 $\pm 290-543$ days) and the overall postoperative infection rate was significantly lower in the autograft group (12.4\%), compared to the allograft cohort (26.3\%).

Present case series showed allogenic bone grafts can be made more effective by adding osteogenic potentiators such as autogenic bone graft and or autologous bone marrow. In our series out of total 18 patients, 4 cases were of delayed union, 9 cases of frank non-union and 5 cases of difficult fractures which had relatively fresh fractures with severe communition and bone loss. Of these 5 cases of difficult fractures, 1 case was open grade II and 2 cases were open grade IIIb. So the biology and soft tissue envelope was not preserved in these cases and there was significant bone loss which required primary bone grafting. Difficult fractures in which primary bone grafting was done took an average time of 7.2 months to unite; the delayed unions took an average time of 8 months to unite while the non unions took a maximum of 12.6 months on an average to unite. Thus relatively older fractures such as in cases of delayed unions and non unions do take a longer time to heal. This appears to signify the need of some osteogenic potentiator along with the allogenic bone graft. These findings are comparable with works of Kong Z et al. [14] who used a combination of bone allograft with autologous red marrow with appropriate internal fixation and reported union rate of $91 \%$ and average time of union of around 4 months for fresh fractures and 6 months for old fractures in their series of 38 patients with varied types of fractures with bone loss (Figure 2). Hierholzer et al. [12] reported an average union time of 4.2 months in case of use of allogenic bone graft to augment plate fixation of humerus fracture non-union's. Similarly Lin WP et al. [15] in 2010 reported a mean union time of 22 weeks in patients with humerus non-union where allogenic bone graft was used to augment locked nailing.

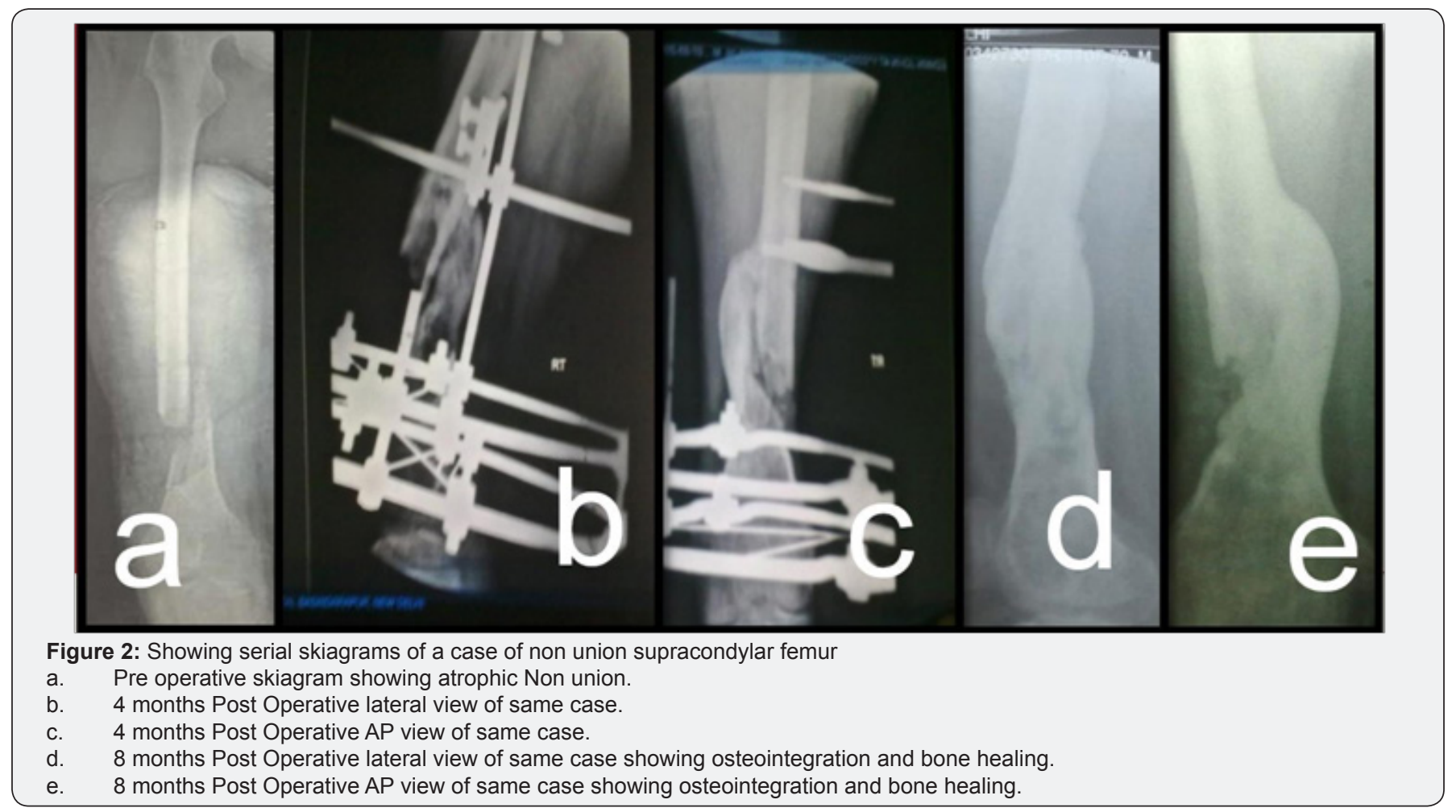

The three failure cases in our series comprised of one established previously infected gap non union of mid diaphysial tibial fracture, one case of delayed union of fracture mid shaft radius and ulna and another of fracture shaft femur. These failure cases belonged to old fractures with hampered healing potential that is delayed/ frank non union; it appears that in such situations use of allogenic bone graft alone not sufficient as some osteogenic potentiator is also required boosting the already slowed healing process (Figure 3). The failure case of tibial fracture non union on subsequent procedure revealed increased vascularity at the fracture site with resorption of allograft and presence of bone defect. These findings are suggestive of allogenic bone graft being able to induce an inflammatory reaction at the fracture site which may aid in healing process but is not sufficient to bring about union as graft resorption occurs at around 4 to 5 months. 


\section{Orthopedics and Rheumatology Open Access Journal (OROAJ)}

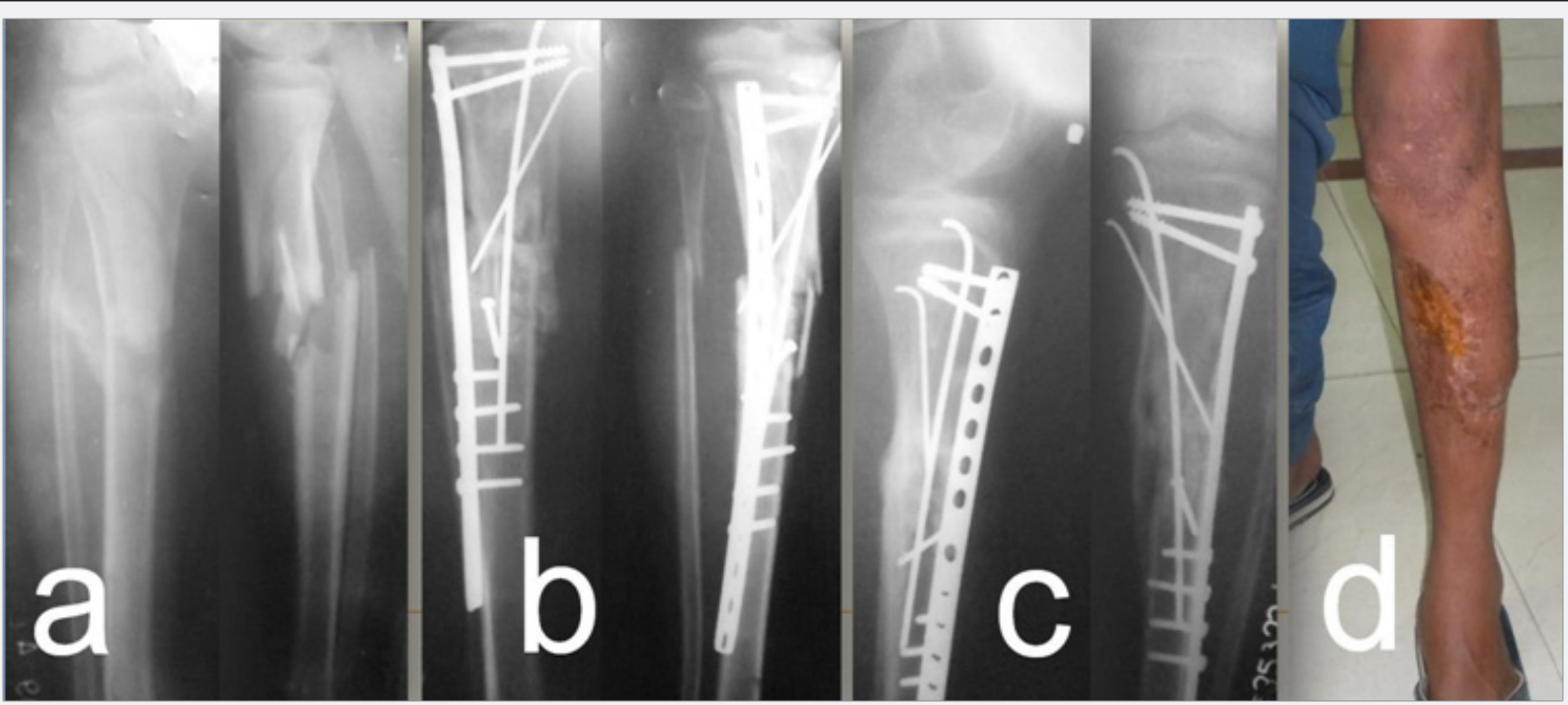

Figure 3: showing serial skiagrams of a case of $30 \mathrm{yr}$ old male with Grade $3 \mathrm{~A}$ compound comminuted proximal tibia fracture.

a. Pre operative AP and lateral views showing extensive comminution.

b. Post operative skiagrams showing surgically managed case with allogenic Bone grafting.

c. 3 month post operative skiagram showing adequate osteo integration and Bone healing.

Allogenic bone grafts are inherentely susceptible to infection as the graft material can serve as foreign body. Infection rates ranging from 4-12\% have been reported by different workers Lord et al. [16] Surgical site infection associated with use of allogenic bone graft shown by Ketonis $\mathrm{C}$ et al. [17] in their study assessing bacteriological colonization of allogenic bone grafts and found a high risk of graft being colonized by microorganisms. Use of allografts in cystic lesions has been found to be associated with increased infection rates (10.9\%) reported in Indian study by Goel SC et al. [18] Use of Vancomycin with allograft had been studied in vitro by Witso E et al. [19] in 1999 and Buttaro MA et al. [20] to prevent infection. Due to above reported complication of infection this study involved the practice of vancomycin impregnation and gamma sterilization of allogenic bone graft. In our series of 18 patients no infection occurred in the post operative period and irrespective of fact that prophylactic vancomycin impregnation was used fractures united and was not found to affect fracture union (Figure 4).
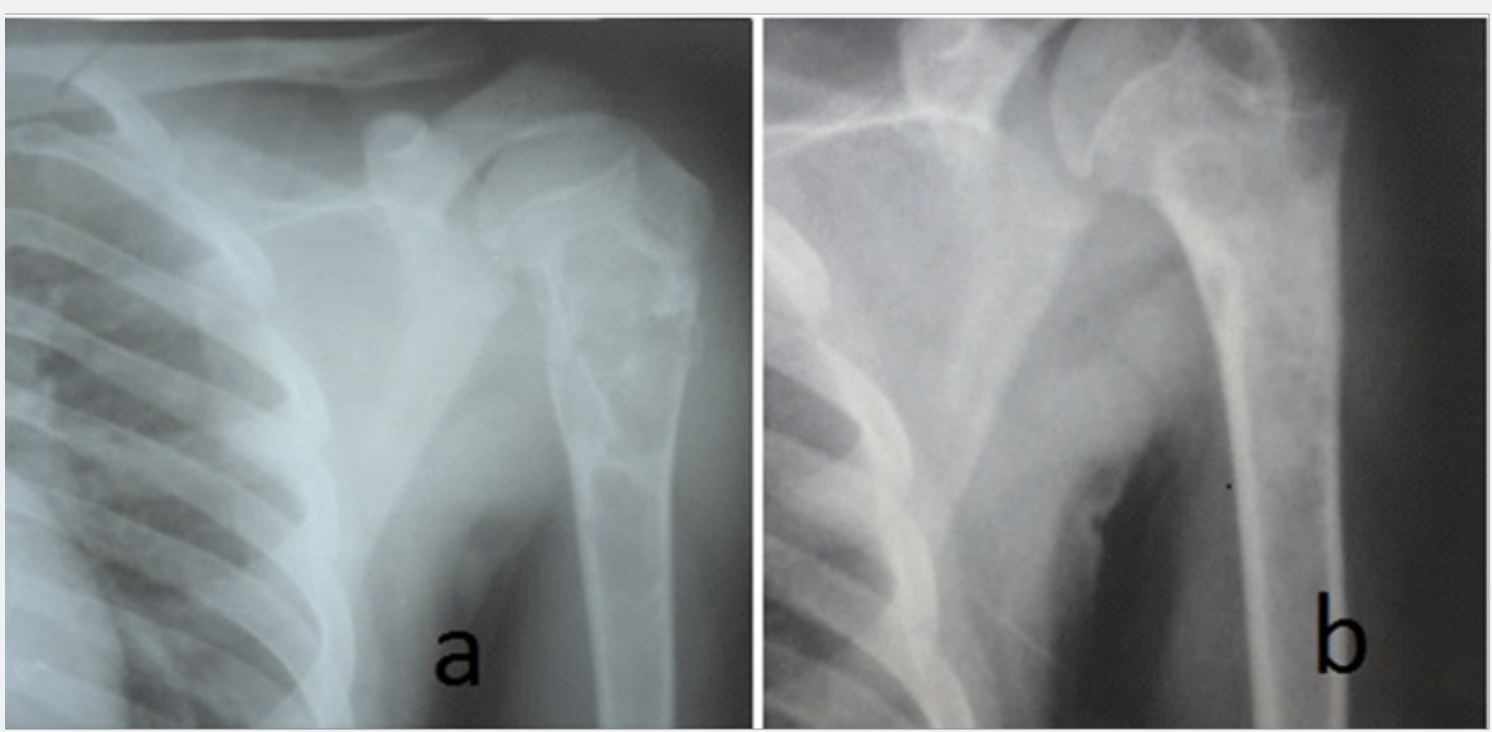

Figure 4: showing serial skiagrams of a case of $14 \mathrm{yr}$ old male with $A B C$ proximal Humerus.

a. Pre Operative AP view showing the cystic lesion.

b. 4 months Post operative view showing adequate osteointegration.

\section{Conclusion}

Allograft bone appears to serve adequate as filler for bony defects. Augmentation of healing in difficult fracture union cases with autologous bone grafts and or bone marrow aspirate appears to be an ideal expander of quantity and benefits of allogenic bone graft to expedited fracture union. Vancomycin impregnation does not appear to hinder fracture healing and graft incorporation process. None of the patients 
showed postoperative infection may be pointing towards use of vancomycin impregnation having benefit of preventing graft related and surgical site infection.

\section{References}

1. Burchardt, Hans (1983) The biology of bone graft repair. Clinical orthopaedics and related research 174: 28-34.

2. Marsh D (1998) Concepts of fracture union, delayed union, and nonunion. Clin Orthop Relat Res 355 (Suppl): S22-S30.

3. Prolo Donald J, Juan J Rodrigo (1985) Contemporary bone graft physiology and surgery. Clinical orthopaedics and related research 200: 322-342.

4. Samartzis D, Shen FH, Goldberg EJ, An HS (2005) Is autograft the gold standard in achieving radiographic fusion in one-level anterior cervical discectomy and fusion with rigid anterior plate fixation? Spine 30(5):1756-1761.

5. Gazdag AR, Lane JM, Glaser D, Forster RA (1995) Alternatives to autogenous bone graft: efficacy and indications. J Am Acad Orthop Surg 3(1): 1-8.

6. Kurz LT, Garfin SR, Booth RE (1989) Harvesting autogenous iliac bone grafts. A review of complications and techniques. Spine 14(12): 13241331.

7. Tomford WW, Mankin HJ (1999) Bone banking: update on methods and materials. Orthop Clin North Am 30(4): 565-570.

8. Burwell RG (1985) The function of bone marrow in the incorporation of a bone graft. Clinical orthopaedics and related research 200: 125141.

9. Ketonis C, Barr S, Adams CS, Hickok NJ, Parvizi J (2010) Bacterial colonization of bone allograft : Establishment and effect of antibiotics. Clin Orthp Rel Res 468(8): 2113-2121.

10. Witso E, Persen L, Benum P, Bergh K (2005) Allogenic decalbone in the repair of benign cystic lesions of bone. Cortical allograft as a vehicle for antibiotic delivery. Acta orthopaedica 76(4): 481-486.

This work is licensed under Creative Commons Attribution 4.0 License

DOI: 10.19080/OROAJ.2018.11.555820
11. Slooff TJ, Huiskes R, van Horn J, Lemmens AJ (1984) Bone grafting in total hip replacement for acetabular protrusion. Acta Orthop Scand 55(6): 593-596.

12. Christian Hierholzer, Domenico Sama, Jose B Toro, Margeret Peterson, David L Helfet (2006) Plate fixation of ununited humeral shaft fractures: effect of type of bone graft on healing. JBJS Volume 88(7): 1442-1447.

13. Flierl MA, Smith WR, Mauffrey C, Irgit K, Williams AE, et al. (2013) Outcomes and complication rates of different bone grafting modalities in long bone fracture nonunions: a retrospective cohort study in 182 patients. J Orthop Surg Res 8:33.

14. Kong Z, Tiang D, Yu H, Feng W, Liu C (2008) Treatment of traumatic bone defect with graft material of allogenic cancellous combined with autologous red marrow. Zhongguo Xiu Fu Chong Jian Wai Ke Za Zhi 22(10): 1251-1254.

15. Lin WP, Lin J (2010) Allografting in Locked Nailing and Interfragmentry Wiring for Humeral Nonunions. Clin Orthop Relat Res 468(3): 852860.

16. Lord CF, Gebhardt MC, Tomford WW, Mankin HJ (1988) Infection in bone allografts. J Bone Joint Surg Am 70: 369-376.

17. Ketonis C, Barr S, Adams CS, Hickok NJ, Parvizi J (2010) Bacterial colonization of bone allograft: Establishment and effect of antibiotics. Clin Orthp Rel Res 468(8): 2113-2121.

18. Goel SC, Tuli SM, Singh HP, Sharma SV, Saraf SK, et al. (1992) 1992;16(2):176-9. Allogenic decalbone in the repair of benign cystic lesions of bone. Int Orthop 16(2): 176-179.

19. Witsø E, Persen L, Løseth K, Bergh K (1999) Adsorption and release of antibiotics from morselized cancellous bone: In vitro studies of 8 antibiotics. Acta Orthop Scand 70(3): 298-304.

20. Buttaro MA, González Della Valle AM, Piñeiro L, Mocetti E, et al. (2003) incorporation of vancomycin-supplemented bone allografts: radiographical, histopathological and immunohistochemical study in pigs. Acta Orthop Scand 74(5): 505-513.

\section{Your next submission with Juniper Publishers will reach you the below assets}

- Quality Editorial service

- Swift Peer Review

- Reprints availability

- E-prints Service

- Manuscript Podcast for convenient understanding

- Global attainment for your research

- Manuscript accessibility in different formats

( Pdf, E-pub, Full Text, Audio)

- Unceasing customer service

Track the below URL for one-step submission https://juniperpublishers.com/online-submission.php 